\title{
Vasohibin-1 as a Novel Prognostic Factor for Head and Neck Squamous Cell Carcinoma
}

\author{
CHISAHO TORII ${ }^{1,2}$, YASUHIRO HIDA ${ }^{3}$, MASANOBU SHINDOH ${ }^{4}$, KOSUKE AKIYAMA $^{1}$, \\ NORITAKA OHGA ${ }^{5}$, NAKO MAISHI $^{1}$, YOICHI OHIRO $^{2}$, MITSUNOBU ONO $^{2}$, YASUNORI TOTSUKA $^{2}$, \\ YOSHIMASA KITAGAWA ${ }^{5}$, $\mathrm{KANCHU} \mathrm{TEI}^{2}$, YASUFUMI SATO ${ }^{6}$ and $\mathrm{KYOKO}^{2}$ HIDA $^{1}$ \\ ${ }^{1}$ Vascular Biology, Frontier Research Unit, Institute for Genetic Medicine, Hokkaido University, Sapporo, Japan; \\ Departments of ${ }^{2}$ Oral and Maxillofacial Surgery, ${ }^{4}$ Oral Pathology and Biology, ${ }^{5}$ Oral Diagnosis and Medicine, \\ Hokkaido University Graduate School of Dental Medicine, Sapporo, Japan; \\ ${ }^{3}$ Department of Cardiovascular and Thoracic Surgery, Hokkaido University \\ Graduate School of Medicine, Sapporo, Japan; \\ ${ }^{6}$ Department of Vascular Biology, Institute of Development, Aging and Cancer, Tohoku University, Miyagi, Japan
}

\begin{abstract}
Aim: We evaluated the prognostic value of vasohibin-1 (VASH1) expression in head and neck squamous cell carcinoma. Materials and Methods: Immunohistochemistry for VASH1 and cluster of differentiation 34 (CD34) was performed on 61 head and neck squamous cell carcinoma specimens. The association between VASH1 expression in the tumour and clinical outcomes was analyzed statistically. Results: VASH1 staining in normal tissue adjacent to cancerous tissue was negative, whereas it was positive in tumour blood vessels and AE1/AE3 and Ki67-positive tumour cells. Therefore, we examined the association between VASHI expression in the tumour and clinical outcomes. Patients with high VASH1 expression in tumour had significantly shorter disease-free survival and more frequently had lymph node recurrence than those with low VASH1 expression. Conclusion: These results suggest that VASH1 expression is associated with tumour progression and may be useful as a prognostic marker of head and neck squamous cell carcinoma.
\end{abstract}

Head and neck squamous cell carcinoma (HNSCC) is the sixth most common cancer in the world (1). HNSCC impairs oral functionality and aesthetics, resulting in a low quality

This article is freely accessible online.

Correspondence to: Dr. Kyoko Hida, Vascular Biology, Frontier Research Unit, Institute for Genetic Medicine, Hokkaido University, N15, W7, Kita-ku, Sapporo 060-0815, Japan. Tel: +81 117064315, Fax: +81 117064325, e-mail: khida@igm.hokudai.ac.jp

Key Words: Angiogenesis, head and neck squamous cell carcinoma, prognosis, vasohibin-1. of life. Tumour stage, lymph node metastasis and invasive pattern contribute to poor outcomes $(2,3)$.

Angiogenesis is the formation of new blood vessels and is important for tumour progression and metastasis (4). Vasohibin-1 (VASH1) is a negative feedback regulator of angiogenesis that is induced by vascular endothelial growth factor (VEGF) and fibroblast growth factor-2 (FGF2) in activated endothelial cells $(5,6)$. Previous studies have shown that VASH1 expression in tumour blood vessels is associated with VEGF and FGF2 expression in tumour cells (7). VASH1 expression in tumour blood vessels is associated with the clinical outcomes of various types of cancers, such as breast cancer, hepatocellular carcinoma, non-small cell lung cancer, prostate cancer, renal cell carcinoma and upper urinary tract urothelial carcinoma (8-15). Conversely, VASH1 expression in tumour cells, but not in tumour blood vessels, is associated with clinical outcomes of colorectal cancer (16).

Here we examined VASH1 expression in HNSCC specimens by immunohistochemistry and investigated whether VASH1 expression was related to clinicopathological factors and prognosis.

\section{Materials and Methods}

Study population. We examined 61 patients with HNSCC who underwent surgical resection at Hokkaido University Hospital, Sapporo, Japan from 1995 to 2008. None of the patients had undergone radiation therapy or chemotherapy preoperatively or postoperatively. Patients were followed-up postoperatively as follows: physical examination every month for the first year, every 2 months for the following year and every 3-6 months for the subsequent years, with CT every 3 months for the first 2 years postoperatively and when relapse was suspected. The follow-up period was 14-194 months (median=71 months). This study was 
approved by the Ethics Committee of Hokkaido University. Patient characteristics are shown in Table I.

Clinical data were retrieved from medical charts. Clinicopathological factors included age, sex, tumour location, tumour stage, $\mathrm{T}$ factor and lymph node metastasis. Stage categories were based on TNM Classification of Malignant Tumours by the Union for International Cancer Control (17).

The normal adjacent tissue apart from the tumour tissue was evaluated as a non-neoplastic counterpart of HNSCC.

Immunohistochemistry. Tissue samples were fixed in $10 \%$ formalin, embedded in paraffin and cut into $4-\mu$ m-thick sections. We performed immunohistochemical staining for VASH1, cluster of differentiation 34 (CD34), pan-cytokeratin with AE1/AE3 as an epithelial marker and for Ki67 as a proliferation marker. Tissue sections were deparaffinized in xylene (Sigma-Aldrich, Tokyo, Japan) and hydrated through a graded alcohol series then finally in distilled water. For antigen retrieval, the tissue sections were heated in a water-bath at $95^{\circ} \mathrm{C}$ for $30 \mathrm{~min}$ in Histofine antigen retrieval solution ( $\mathrm{pH}$ 9.0; Nichirei Corporation, Tokyo, Japan) diluted at $1: 10$. Endogenous peroxidase activity was blocked by incubation in $0.5 \%$ hydrogen peroxidase (Sigma-Aldrich) for $10 \mathrm{~min}$ at room temperature. Then the sections were incubated with a blocking solution of 5\% goat serum in tris-buffered saline (TBS) for $30 \mathrm{~min}$.

The processed sections were stained overnight at $4{ }^{\circ} \mathrm{C}$ with primary monoclonal antibodies. The primary antibodies used were as follows: mouse monoclonal anti-human VASH1 (diluted at 1:1000), rabbit polyclonal anti-human CD34 (EP373Y, diluted at 1:500; Abcam, Cambridge, MA, USA), mouse monoclonal antihuman cytokeratin, clone AE1/AE3 (IR053, diluted at 1:20; Dako, Tokyo, Japan) and mouse anti-human Ki-67 (M7240, diluted at 1:500; Dako). The specificity of this antibody to VASH1 was documented in a previous study (5). After washing with TBS, the sections were incubated with secondary antibodies for $60 \mathrm{~min}$ at room temperature. 3,3'-Diaminobenzidine was used as the chromogen (K3468, Liquid DAB+ Substrate Chromogen System; Dako). The sections were then counterstained with hematoxylin (Wako, Osaka, Japan).

Evaluation of immunohistochemical staining. All sections were independently evaluated by two investigators. When the results of the evaluations were different, another investigator performed an evaluation for the final decision. All investigators were blinded to the clinicopathological information. To analyse VASH1 expression in the tumour, as many areas as possible were randomly selected within the tumour at low magnification $(\times 100)$. The number of areas observed depended on the tumour sample size. The pixel number in the VASH1-positive area at high magnification $(\times 200)$ was determined using ImageJ software (National Institutes of Health, Bethesda, MD, USA). VASH1 expression in the tumour was calculated as the ratio of the pixel number of the VASH1-positive area to that of the total area. The cut-off for VASH1 expression in the tumour was $1.1 \%$ for the analysis of disease-free survival, which was the point with the lowest $p$-value in a log-rank test.

Statistical analysis. The association between clinicopathological factors and VASH1 expression in the tumour was validated by chi-square tests. We also used univariate and multivariate analyses with Cox proportional hazards regression model. Disease-free survival and lymph node recurrence were estimated using the Kaplan-Meier method and analysed by the log-rank test. Differences among groups were regarded as significant when $p<0.05$. Statistical analyses were performed using SPSS version 18.0 (IBM Corporation, Armonk, NY, USA).

\section{Results}

VASH1 expression in HNSCC and patient characteristics. Blood vessels in HNSCC specimens and normal adjacent tissue were CD34-positive (Figure 1A, C, E and G). Although the normal adjacent tissue was VASH1-negative (Figure 1B), tumour blood vessels were VASH1-positive (Figure 1D); however, some cases were VASH1-negative (Figure 1F). Not only tumour blood vessels but also tumour cells were VASH1-positive (Figure $1 \mathrm{H}$ ). We confirmed that VASH1-positive cells, except for tumour endothelial cells, were tumour cells by immunohistochemistry for AE1/AE3 as an epithelial marker. HNSCC cells were AE1/AE3positive, and serial sections showed that some AE1/AE3positive tumour cells were also VASH1-positive (Figure 2AD). Some VASH1-positive tumour cells appeared to be Ki67positive (Figure 2E and F). These results indicate that some VASH1-positive tumour cells were proliferative.

Table I indicates the clinicopathological parameters of 61 patients. Among 61 cases, eight patients died (three with HNSCC, five from, other causes). Nine patients developed lymph node recurrence out of the 12 patients who experienced relapse. The mean and median duration of disease-free survival was 56.2 and 59 months, respectively.

Prognostic significance of VASH1 expression in patients with HNSCC. The cut-off for VASH1 expression in the tumour for survival analyses was $1.1 \%$. Cases with $<1.1 \%$ VASH 1 expression in tumour were defined as having low VASH1 expression and the remaining cases were defined as having high VASH1 expression. Thirty-one cases had high VASH1 expression (Table I). Univariate analysis revealed that lymph node metastasis and VASH1 expression in the tumour were significant predictors of recurrence $(p=0.0457$ and 0.0367 , respectively). Multivariate analysis revealed that VASH1 expression in the tumour was a significant predictor of recurrence $(p=0.045$, hazard ratio $=3.04)$. Multivariate analysis confirmed that high VASH1 expression in the tumour was an independent predictor of poorer disease-free survival.

Table II shows the association between VASH1 expression level in the tumour and clinicopathological parameters in 61 patients with HNSCC. High VASH1 expression in the tumour was significantly associated with lymph node recurrence $(p=0.018)$. Disease-free survival was significantly lower in cases with high VASH1 expression in the tumour $(p=0.027$, Figure 3$)$. Sixty percent of relapses were lymph node recurrence. These results indicated that high VASH1 expression was an independent predictor of lymph node recurrence and of poorer disease-free survival of HNSCC. 
Table I. Univariate and multivariate analysis for disease-free survival in 61 patients with head and neck squamous cell carcinoma.

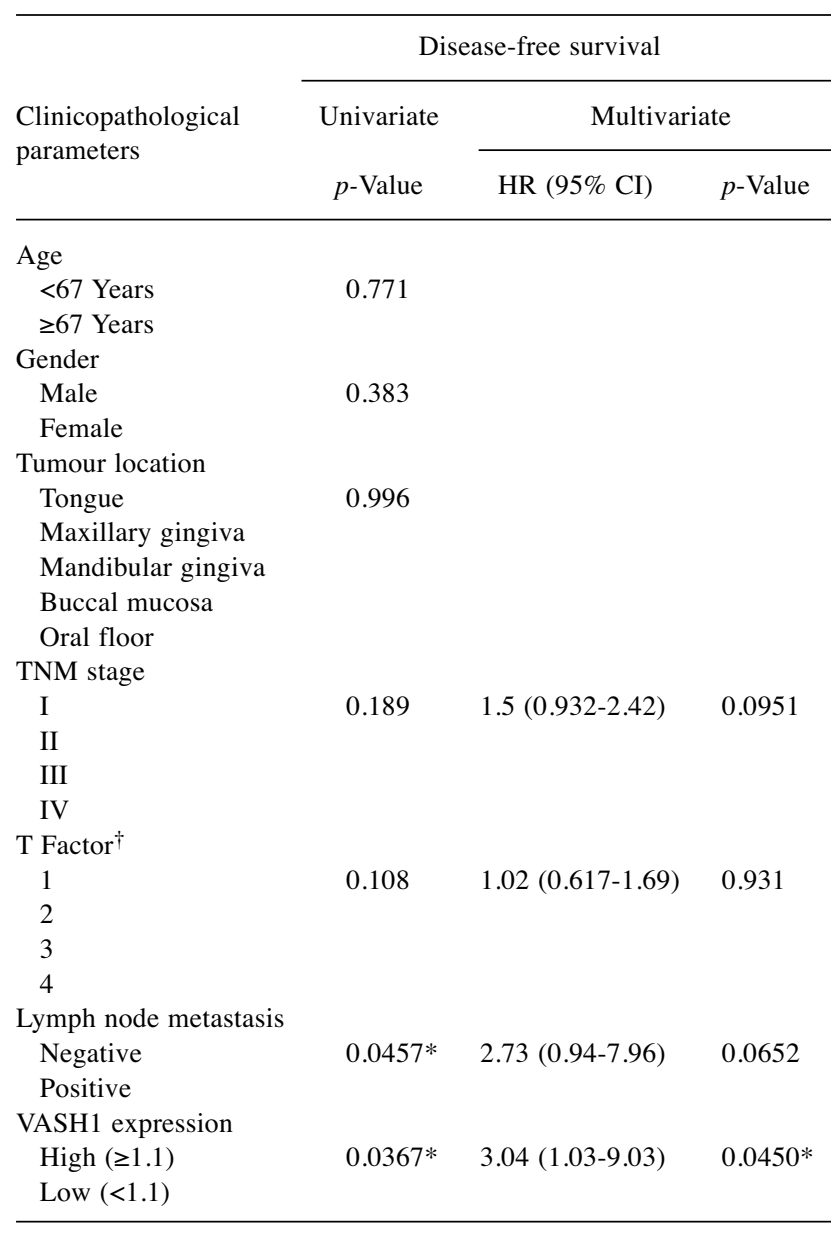

HR: Hazard ratio: CI: confidence interval; VASH1: vasohibin-1. ${ }^{\dagger}$ According to the sixth edition of TNM staging guidelines (17). $* p<0.05$ was considered statistically significant.

\section{Discussion}

Here we revealed that HNSCC tumour and vascular endothelial cells expressed VASH1. We also found that high VASH1 expression in HNSCC was significantly associated with shorter disease-free survival and higher lymph node recurrence. Our study shows, as far as we are aware for the first time, that VASH1 is a prognostic factor for HNSCC.

Recently, associations have been reported between VASH1 expression and prognosis of patients with various types of cancer (8-15). For example, in prostate cancer and non-small cell lung cancer, VASH1 expression was associated with tumour progression, and high VASH1 expression was a negative predictor, with specific VASH1 expression in tumour-associated endothelial cells being reported $(11,12)$.
Table II. Clinicopathological parameters in 61 patients according to vasohibin-1 (VASH1) expression.

\begin{tabular}{|c|c|c|c|}
\hline \multirow[b]{2}{*}{$\begin{array}{l}\text { Clinicopathological } \\
\text { parameters }\end{array}$} & \multicolumn{2}{|c|}{ No of patients (\%) } & \multirow[b]{2}{*}{$p$-Value } \\
\hline & $\begin{array}{l}\text { Low VASH1 } \\
(\mathrm{n}=30)\end{array}$ & $\begin{array}{l}\text { High VASH1 } \\
\quad(n=31)\end{array}$ & \\
\hline \multicolumn{4}{|l|}{ Age } \\
\hline$<67$ Years & $15(50.0)$ & $14(45.2)$ & 0.705 \\
\hline$\geq 67$ Years & $15(50.0)$ & $17(54.8)$ & \\
\hline \multicolumn{4}{|l|}{ Gender } \\
\hline Male & $16(53.3)$ & $18(58.1)$ & 0.71 \\
\hline Female & $14(46.7)$ & $13(41.9)$ & \\
\hline \multicolumn{4}{|l|}{ Tumour location } \\
\hline Tongue & $11(36.7)$ & $11(35.5)$ & 0.472 \\
\hline Maxillary gingiva & $6(20.0)$ & $3(9.68)$ & \\
\hline Mandibular gingiva & $6(20.0)$ & $8(25.8)$ & \\
\hline Buccal mucosa & $3(10.0)$ & $7(22.6)$ & \\
\hline Oral floor & $4(13.3)$ & $2(6.45)$ & \\
\hline \multicolumn{4}{|l|}{ TNM stage } \\
\hline I & $9(30.0)$ & $8(25.8)$ & 0.509 \\
\hline II & $11(36.7)$ & $7(22.6)$ & \\
\hline III & $5(16.7)$ & $8(25.8)$ & \\
\hline IV & $5(16.7)$ & $8(25.8)$ & \\
\hline \multicolumn{4}{|l|}{ T Factor ${ }^{\dagger}$} \\
\hline 1 & $10(33.3)$ & $8(25.8)$ & 0.322 \\
\hline 2 & $14(46.7)$ & $10(32.3)$ & \\
\hline 3 & $2(6.7)$ & $5(16.1)$ & \\
\hline 4 & $4(13.3)$ & $8(25.8)$ & \\
\hline \multicolumn{4}{|l|}{ Lymph node metastasis } \\
\hline Negative & $26(84.4)$ & $23(75.9)$ & 0.221 \\
\hline Positive & $4(12.5)$ & $8(13.8)$ & \\
\hline \multicolumn{4}{|l|}{ Local recurrence } \\
\hline Negative & $26(86.7)$ & $27(74.2)$ & 0.96 \\
\hline Positive & $4(13.3)$ & $4(12.9)$ & \\
\hline \multicolumn{4}{|l|}{ Lymph node recurrence } \\
\hline Negative & $27(90.0)$ & $20(64.5)$ & $0.018^{*}$ \\
\hline Positive & $3(10.0)$ & $11(35.5)$ & \\
\hline
\end{tabular}

$\dagger$ According to the sixth edition of TNM staging guidelines (17). $* p<0.05$ was considered statistically significant.

VASH1 expression in tumour cells has been reported in renal cell cancer and colon cancer $(13,16,18)$. Yan et al. reported a strong positive association between VASH1 and VEGFA expression and microvessel density (MVD) in colorectal cancer tissues. VASH1 expression was significantly positively associated with pathological TNM stage, tumour stromal invasion, lymph node involvement, distant metastasis and shorter survival (16). VASH1 expression was reported in colon cancer cells and this was positively associated with tumour progression, MVD and poor prognosis (18). In contrast to these studies on colon cancer, Zhao et al. reported that higher VASH1 expression was associated with a lower MVD and better prognosis of renal cell cancer (13). Such differences among different cancer types might be attributable to observations of constitutive 


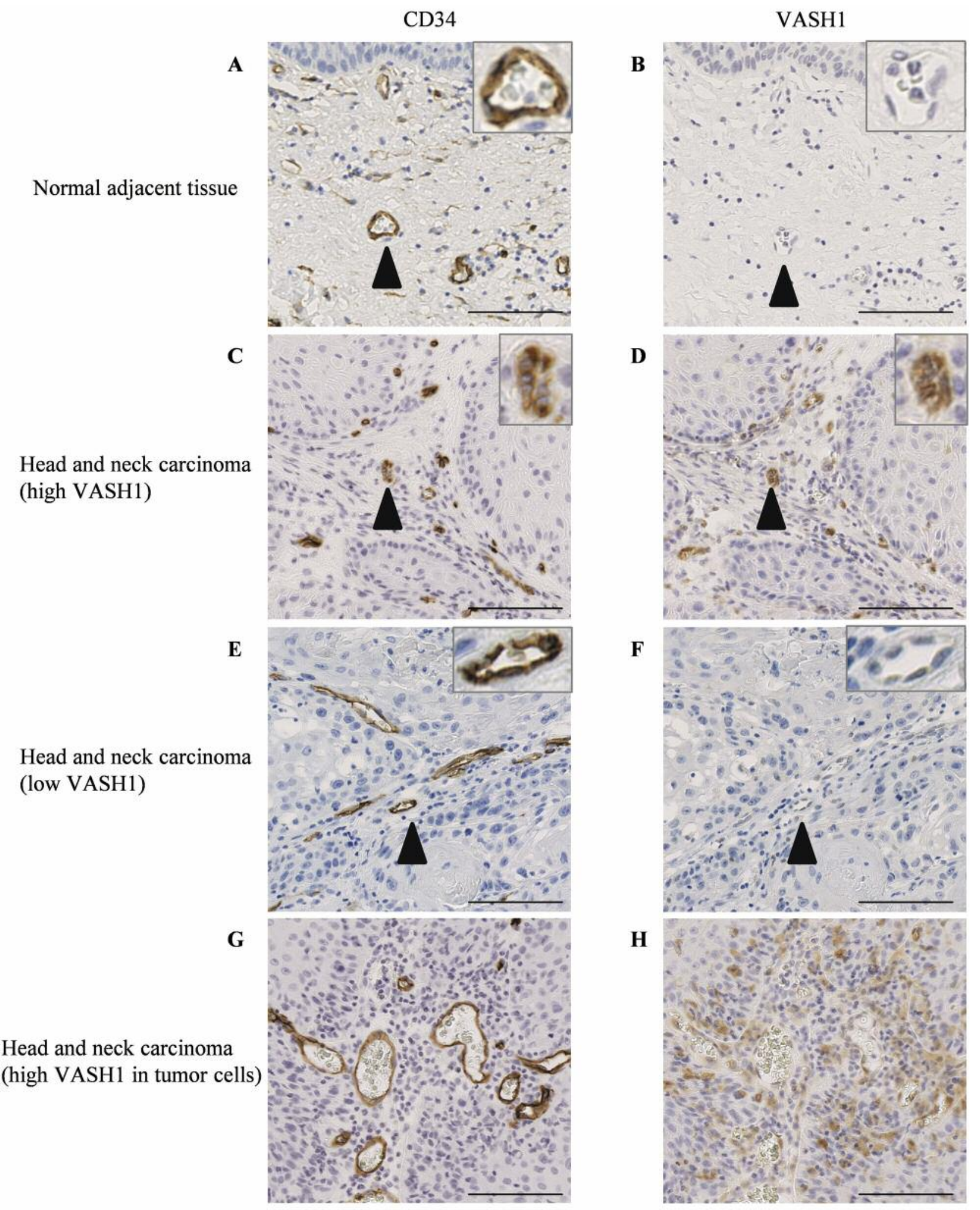

Figure 1. Immunostaining of cluster of differentiation 34 (CD34) and vasohibin-1 (VASH1) in head and neck squamous cell carcinoma and normal adjacent tissue. CD34 staining in both normal blood vessels $(A)$ and tumour blood vessels $(C, E$ and $G)$ was positive. VASH1 staining in normal adjacent tissue was negative (B). VASH1 staining in tumour blood vessels was high (D) but also low $(F)$. VASH1 staining was high in tumour cells $(H)$. Insets are enlarged images of the regions indicated by the arrowheads. Scale bar $=0.1 \mathrm{~mm}$. 


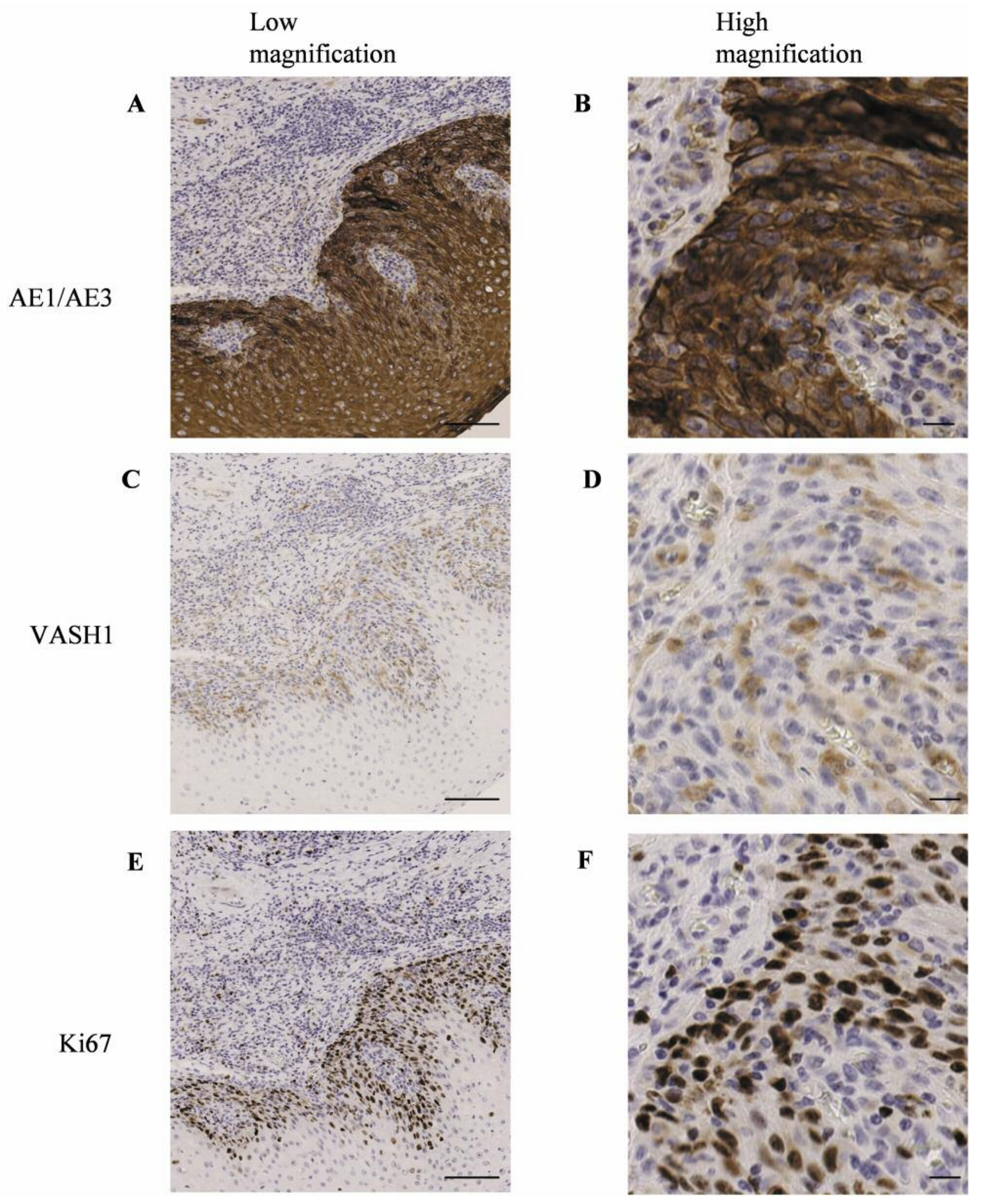

Figure 2. Immunostaining of serial sections stained with pan-cytokeratin antibody AE1/AE3 as a marker of epithelial cells (A and B), antibody to vasohibin-1 (VASH1) ( $C$ and D) and antibody Ki67 as a marker of proliferation ( $E$ and $F$ ) in head and neck squamous cell carcinoma. AE1/AE3 staining was positive in tumour cells (A and B). VASH1 staining was also positive in tumour cells (C and D). Ki-67 staining was positive in VASH1positive tumour cells ( $E$ and $F$ ). Scale bar $=0.1 \mathrm{~mm}, A, C$ and $E$; scale bar $=0.01 \mathrm{~mm}, B, D$ and $F$. 


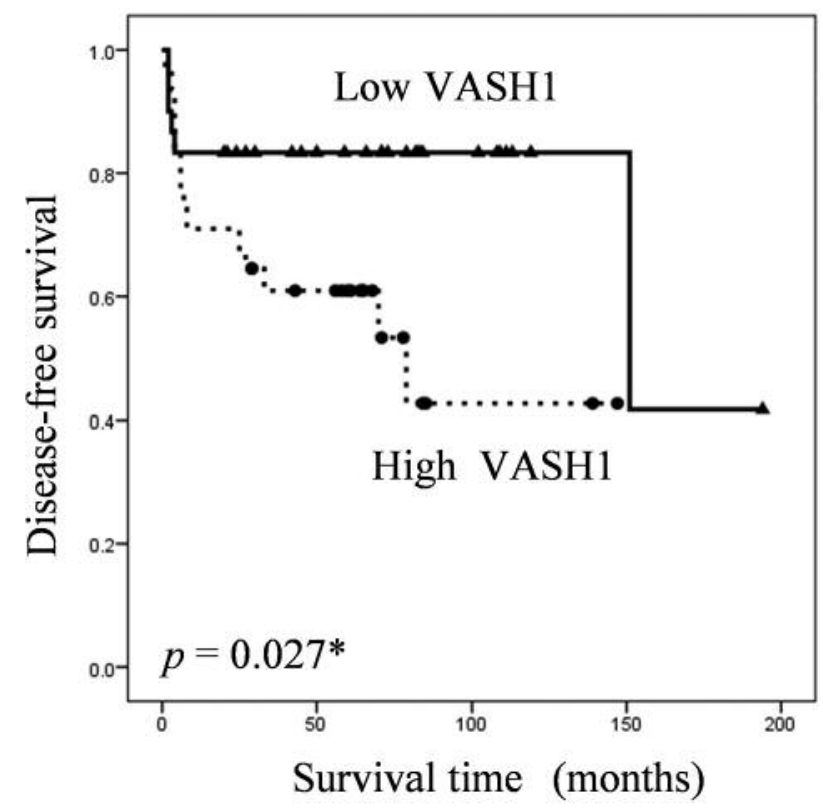

Figure 3. Kaplan-Meier curve of disease-free survival according to vasohibin-1 (VASH1) expression in head and neck squamous cell carcinoma. ${ }^{*} p<0.05$ was considered statistically significant.

VASH1 expression in the adjacent normal tissue in the kidney, but not in the colon. There may be different types of regulation and roles of VASH1 in each organ. Our immunohistochemical analyses of HNSCC specimens confirmed VASH1 expression in tumour and endothelial cells, while no VASH1 expression was found in the normal adjacent tissue. The fact that VASH1-positive tumour cells were also positive for Ki67 suggests that these tumour cells were proliferative. The association between Ki67 and VASH1 in tumour cells has not been reported; VASH1 expression itself might promote tumour development or merely reflect VEGFA abundance in the tissue.

HNSCC negative for cervical node involvement, as diagnosed by palpation, computed tomography and $2-\left[{ }^{18} \mathrm{~F}\right]$ fluoro-2-deoxy-D-glucose positron-emission tomography, may in fact have micrometastasis in the lymph node. It is estimated that the prevalence lymph node micrometastasis is $20-40 \%$ (19). Patients with lymph node micrometastasis will likely develop lymph node metastases in a few months to several years. Therefore, biomarkers capable of predicting lymph node recurrence would be useful in determining whether cervical lymph node dissection is necessary.

Our study showed that VASH1 expression is a predictor of shorter disease-free survival, as well as highlighting an association between high VASH1 expression and lymph node recurrence. These results suggest that VASH1 expression in preoperative biopsy specimens might be a useful marker for determining treatment and follow-up strategies. A larger cohort study would clarify the importance of VASH1 expression in HNSCC tumour.

Our study showed that VASH1 is expressed in HNSCC tumour and tumour endothelial cells and that VASH1 expression is associated with poorer disease-free survival and lymph node recurrence.

\section{Acknowledgements}

The Authors thank Drs. Takayuki Hojo, Kenji Yamada, Taisuke Kawamoto and Ms. Yuko Suzuki for their technical assistance.

\section{References}

1 Leemans CR, Braakhuis BJ and Brakenhoff RH: The molecular biology of head and neck cancer. Nat Rev Cancer 11: 9-22, 2011.

2 Chang YC, Nieh S, Chen SF, Jao SW, Lin YL and Fu E: Invasive pattern grading score designed as an independent prognostic indicator in oral squamous cell carcinoma. Histopathology 57: 295-303, 2010.

3 Kim SY, Nam SY, Choi SH, Cho KJ and Roh JL: Prognostic value of lymph node density in node-positive patients with oral squamous cell carcinoma. Ann Surg Oncol 18: 2310-2317, 2011.

4 Folkman J: Angiogenesis in cancer, vascular, rheumatoid and other disease. Nat Med 1: 27-31, 1995.

5 Watanabe K, Hasegawa Y, Yamashita H, Shimizu K, Ding Y, Abe M, Ohta H, Imagawa K, Hojo K, Maki H, Sonoda H and Sato Y: Vasohibin as an endothelium-derived negative feedback regulator of angiogenesis. J Clin Invest 114: 898-907, 2004.

6 Kimura H, Miyashita H, Suzuki Y, Kobayashi M, Watanabe K, Sonoda H, Ohta H, Fujiwara T, Shimosegawa T and Sato Y: Distinctive localization and opposed roles of vasohibin-1 and vasohibin-2 in the regulation of angiogenesis. Blood 113: 48104818, 2009.

7 Hosaka T, Kimura H, Heishi T, Suzuki Y, Miyashita H, Ohta H, Sonoda H, Moriya T, Suzuki S, Kondo T and Sato Y: Vasohibin1 expression in endothelium of tumour blood vessels regulates angiogenesis. Am J Pathol 175: 430-439, 2009.

8 Tamaki K, Moriya T, Sato Y, Ishida T, Maruo Y, Yoshinaga K, Ohuchi $\mathrm{N}$ and Sasano $\mathrm{H}$ : Vasohibin-1 in human breast carcinoma: a potential negative feedback regulator of angiogenesis. Cancer Science 100: 88-94, 2009.

9 Tamaki K, Sasano H, Maruo Y, Takahashi Y, Miyashita M, Moriya T, Sato Y, Hirakawa H, Tamaki N, Watanabe M, Ishida $\mathrm{T}$ and Ohuchi $\mathrm{N}$ : Vasohibin-1 as a potential predictor of aggressive behaviour of ductal carcinoma in situ of the breast. Cancer Science 101: 1051-1058, 2010.

10 Murakami K, Kasajima A, Kawagishi N, Sekiguchi S, Fujishima F, Watanabe M, Sato Y, Ohuchi N and Sasano H: The prognostic significance of vasohibin 1-associated angiogenesis in patients with hepatocellular carcinoma. Hum Pathol 45: 589-597, 2014.

11 Zhang T, Yu TT, Zhang DM, Hou XM, Liu XJ, Zhao D and Shan L: Vasohibin-1 expression detected by immunohistochemistry correlates with prognosis in non-small cell lung cancer. Med Oncol 31: 963, 2014.

12 Kosaka T, Miyazaki Y, Miyajima A, Mikami S, Hayashi Y, Tanaka N, Nagata H, Kikuchi E, Nakagawa K, Okada Y, Sato Y 
and Oya M: The prognostic significance of vasohibin-1 expression in patients with prostate cancer. Br J Cancer 108: 2123-2129, 2013.

13 Zhao G, Yang Y, Tang Y, Han R and Sun Y: Reduced expression of vasohibin-1 is associated with clinicopathological features in renal cell carcinoma. Med Oncol 29: 3325-3334, 2012.

14 Kanomata N, Sato Y, Miyaji Y, Nagai A and Moriya T: Vasohibin-1 is a new predictor of disease-free survival in operated patients with renal cell carcinoma. J Clin Pathol 66: 613-619, 2013.

15 Miyazaki Y, Kosaka T, Mikami S, Kikuchi E, Tanaka N, Maeda T, Ishida M, Miyajima A, Nakagawa K, Okada Y, Sato Y and Oya M: The prognostic significance of vasohibin-1 expression in patients with upper urinary tract urothelial carcinoma. Clin Cancer Res 18: 4145-4153, 2012.

16 Yan Y, Shen Z, Ye Y, Jiang K, Zhang H, Shen C, Mustonen H, Puolakkainen $\mathrm{P}$ and Wang S: A novel molecular marker of prognosis in colorectal cancer: Vasohibin-1. Med Oncol 31: 816, 2014.
17 International Union against Cancer: TNM: Classification of Malignant Tumours. Sobin LH and Wittekind CI (eds.). New York: Wiley-Liss, 2002.

18 Kitajima T, Toiyama Y, Tanaka K, Saigusa S, Kobayashi M, Inoue Y, Mohri Y and Kusunoki M: Vasohibin-1 increases the malignant potential of colorectal cancer and is a biomarker of poor prognosis. Anticancer Res 34: 5321-5329, 2014.

19 Greenberg JS, Fowler R, Gomez J, Mo V, Roberts D, El Naggar AK and Myers JN: Extent of extracapsular spread: a critical prognosticator in oral tongue cancer. Cancer 97: 1464-1470, 2003.

Received December 15, 2016

Revised February 6, 2017

Accepted February 7, 2017 